\title{
The mitochondrial genome and Epigenome of the Golden lion Tamarin from fecal DNA using Nanopore adaptive sequencing
}

\author{
Nicole Wanner ${ }^{1}$, Peter A. Larsen², Adam McLain ${ }^{3}$ and Christopher Faulk ${ }^{*}$
}

\begin{abstract}
Background: The golden lion tamarin (Leontopithecus rosalia) is an endangered Platyrrhine primate endemic to the Atlantic coastal forests of Brazil. Despite ongoing conservation efforts, genetic data on this species remains scarce. Complicating factors include limitations on sample collection and a lack of high-quality reference sequences. Here, we used nanopore adaptive sampling to resequence the L. rosalia mitogenome from feces, a sample which can be collected non-invasively.

Results: Adaptive sampling doubled the fraction of both host-derived and mitochondrial sequences compared to sequencing without enrichment. 258x coverage of the L. rosalia mitogenome was achieved in a single flow cell by targeting the unfinished genome of the distantly related emperor tamarin (Saguinus imperator) and the mitogenome of the closely related black lion tamarin (Leontopithecus chrysopygus). The L. rosalia mitogenome has a length of $16,597 \mathrm{bp}$, sharing $99.68 \%$ sequence identity with the L. chrysopygus mitogenome. A total of 38 SNPs between them were identified, with the majority being found in the non-coding D-loop region. DNA methylation and hydroxymethylation were directly detected using a neural network model applied to the raw signal from the MinION sequencer. In contrast to prior reports, DNA methylation was negligible in mitochondria in both CpG and non-CpG contexts. Surprisingly, a quarter of the $642 \mathrm{CpG}$ sites exhibited DNA hydroxymethylation greater than 1\% and 44 sites were above $5 \%$, with concentration in the $3^{\prime}$ side of several coding regions.
\end{abstract}

Conclusions: Overall, we report a robust new mitogenome assembly for L. rosalia and direct detection of cytosine base modifications in all contexts.

Keywords: Mitochondria, DNA methylation, DNA hydroxymethylation, Poop, Primates, Lion Tamarin

\section{Introduction}

The golden lion tamarin (Leontopithecus rosalia) is an endangered Platyrrhine primate endemic to the Atlantic coastal forests of Brazil [1]. It is a member of the family Callitrichidae, a taxonomic grouping that includes marmosets, tamarins and lion tamarins [2]. Although once teetering on the brink of extinction, golden lion tamarins

\footnotetext{
* Correspondence: cfaulk@umn.edu

'Department of Animal Sciences, University of Minnesota, College of Food, Agricultural, and Natural Resource Sciences, 1988 Fitch Ave., Saint Paul, MN 55108, USA

Full list of author information is available at the end of the article
}

have benefitted from a successful captive breeding and reintroduction program that has seen their numbers climb from a few hundred individuals in the wild to several thousand. In addition to wild individuals, several hundred animals are maintained in captivity globally as part of the captive breeding and reintroduction program, and stud books are maintained to promote genetic diversity in these animals and avoid inbreeding [3]. Golden lion tamarins are gregarious, living in social groups typically centered around a monogamous breeding pair and their dependent infants, juveniles and subadults [4]. Golden lion tamarins also play an important ecological

(c) The Author(s). 2021 Open Access This article is licensed under a Creative Commons Attribution 4.0 International License, which permits use, sharing, adaptation, distribution and reproduction in any medium or format, as long as you give appropriate credit to the original author(s) and the source, provide a link to the Creative Commons licence, and indicate if changes were made. The images or other third party material in this article are included in the article's Creative Commons licence, unless indicated otherwise in a credit line to the material. If material is not included in the article's Creative Commons licence and your intended use is not permitted by statutory regulation or exceeds the permitted use, you will need to obtain permission directly from the copyright holder. To view a copy of this licence, visit http://creativecommons.org/licenses/by/4.0/. The Creative Commons Public Domain Dedication waiver (http://creativecommons.org/publicdomain/zero/1.0/) applies to the data made available in this article, unless otherwise stated in a credit line to the data. 
role as seed dispersers [5]. Genetic data from L. rosalia remains scant, however. Increased availability of genetic data will benefit breeding and conservation efforts for this species.

Mitochondria are the powerhouse of the cell and contain their own genome [6]. The mitogenome in animals is small, circular, and mutates more rapidly than the nuclear genome. Two characteristics make it especially useful for phylogenetic comparisons and non-invasive sampling. First, it is present in many more copies per cell than the nuclear genome, making it easier to recover from degraded samples such as feces or ancient DNA. Second, its higher mutation rate enables more accurate delineation of closely related species. The first mitochondrial sequences of $L$. rosalia were published in 2008 and 2011 and were limited to the sequences of cytochrome $\mathrm{b}$ and the hypervariable region of the displacement control region (D-loop) [7, 8]. A more complete version of the mitogenome became available in 2013, but it contained gaps and shared surprisingly limited homology with the closely related black lion tamarin $(L$. chrysopygus) with only $96 \%$ shared identity [9]. To create a more accurate comparison of the L. rosalia mitogenome to its sister species, we chose to resequence its mitochondrial genome to high coverage using a novel technique with easily collected fecal samples.

Nanopore sequencing is based on electrical signals generated by cylindrical protein pores as nucleic acids pass through [10]. The MinION sequencer from Oxford Nanopore Technologies (ONT) is field-portable with minimal reagent requirements. It can provide long reads with an average read length of $20 \mathrm{~kb}$ and occasional single reads over $1 \mathrm{Mb}$ in length, limited only by the molecular weight of the input DNA. Since mitochondria have $\sim 16 \mathrm{~kb}$ genomes, read lengths are sufficient to cover the entire mitogenome. However, in fecal samples, the mixture of DNA sources presents a challenge to sequencing.

Traditionally, targeted sequencing of specific loci is performed by enzymatic or PCR enrichment prior to sequencing [11]. Recently, a form of computational enrichment called adaptive sampling has been developed [12]. This method allows for sequencing of whole genomic DNA combined with locus-specific enrichment by rejecting off target reads. Crucially, adaptive sampling can enrich target regions up to 30 -fold, dependent on sequence length and percentage of the genome, enough to bring target loci to high enough coverage for analysis. Here, we employed adaptive sampling in order to obtain sufficient coverage of the mitogenome for accurate assembly.

From an epigenetic perspective, mitochondrial DNA methylation has been a matter of debate [13]. Determining its methylation pattern has been challenging due to unique characteristics including resistance to bisulfite transformation, which is used in nearly all epigenetic methods [14]. Direct sequencing of native genomic DNA preserves base modifications which can then be detected with nanopore sequencing. Neural network models are capable of providing simultaneous calls of nucleic acid sequence and base modifications from unenriched genomic DNA input [15]. Here we focus on 5'methylcytosine at both $\mathrm{CpG}$ and non-CpG sites. Other DNA modifications such as $6 \mathrm{~N}$-adenine $(\mathrm{m} 6 \mathrm{~A})$ are present in the human genome and mitochondria $[13,16]$. Theoretically, m6A could be detected from the raw signal by future neural network models, however, none are currently able to accurately detect it. Due to the recent availability of models for calling DNA 5'hydroxymethylcytosine, we are the first to report native detection at base-pair resolution in mitochondria by sequencing. The combination of adaptive sampling, long-reads, and epigenetic information, drove our selection of the Oxford Nanopore MinION to resequence the mitogenome of $L$. rosalia.

Fecal DNA serves as a rich source of host information that can be collected non-invasively from wild populations and processed for DNA extraction in the field [17]. Fecal microbiomes from nanopore sequencing have been the subject of multiple studies [18-20], however, host DNA enrichment by nanopore sequencing from fecal samples has not yet been documented. Due to degradation of DNA by digestive processes, choosing high abundance targets such as mitochondria naturally increases their sequencing frequency at the cost of sequence read length.

We describe an improved assembly of the mitogenome of the golden lion tamarin extracted from a fecal sample. We find that this species is most closely related to the black lion tamarin based on the high level of sequence identity. This finding is consistent with prior taxonomic studies using genetics and morphology, which found the two species to be closely related [7, 21, 22]. We confirm the pattern of diverging mutations falls mostly within the non-coding D-loop. Our method resulted in $285 \mathrm{x}$ coverage from a single flow cell. The use of a high accuracy' neural network model with $97.8 \%$ modal accuracy in base calling allows high confidence in the consensus. Sequence polishing, error-correcting, and assembly resulted in a circular contig of $16,597 \mathrm{bp}$ in length. We directly measured DNA methylation and hydroxymethylation levels from the nanopore signal level read data using the Megalodon tool provided by ONT. We find that DNA methylation is negligible across the entire mitogenome, in line with human mtDNA [6]. Surprisingly, we find elevated levels of DNA hydroxymethylation only in the $\mathrm{CpG}$ context, suggesting biological function of this mark in mitochondria. We 
conclude that fecal samples provide a rich source of host DNA suitable for nanopore sequencing, providing a new capability for field use in conservation research.

\section{Results}

Fecal DNA yields a large quantity of highly fragmented DNA

Wild-collected feces represent an abundant supply of host genomic, metabolomic, and metagenomic information. We collected a fresh, wet fecal sample from a captive golden lion tamarin at the Utica Zoo. Two kit-based methods of DNA extraction were used with manufacturer protocols designed to enrich the host DNA fraction and yielded similar results. A total of $9 \mu \mathrm{g}$ of DNA was derived from $400 \mathrm{mg}$ of fecal material and used for library prep and nanopore sequencing. Library prep with $2 \mu \mathrm{g}$ input yielded between 200 and $700 \mathrm{ng}$ of product per reaction. Mean read length from untargeted nanopore sequencing was $1230 \mathrm{bp}$, indicating short fragment length likely due to digestive degradation.

\section{Nanopore adaptive sampling enriches host DNA from feces}

A nanopore MinION flowcell was loaded with $500 \mathrm{ng}$ of DNA and sequenced for $24 \mathrm{~h}$. Since over $99 \%$ of fecal DNA is derived from microbiome and digesta, enriching the small fraction of host-derived fragments makes better use of limited sequencing capacity. We used adaptive sampling to enrich for sequences that matched to the unfinished genome of a distantly related species, Saguinus imperator (emperor tamarin). This target genome consists of 3.4 Gb total sequence divided into 1,666,189 scaffolds. S. imperator was chosen over the more closely related Leontopithecus chrysopygus (black lion tamarin) since the latter did not have an available nuclear genome.

Our initial sequencing run yielded 15 million reads containing $6.3 \mathrm{~Gb}$ of sequence with a mean read length of $411 \mathrm{bp}$, indicating that adaptive sampling was rejecting most reads in less than $1 \mathrm{~s}$ since the read rate averaged over 400 bases/s. All base-called sequence was kept, whether or not it is from a rejected strand, and used for analysis. We identified $0.8 \%$ of reads matching $S$. imperator, derived from the fecal sample of $L$. rosalia DNA, compared to $0.39 \%$ without enrichment, therefore adaptive sampling was successful in enriching host tamarin sequences (Table 1).

\section{Mapping mitochondrial reads}

To identify mitochondrially derived reads, we initially mapped the total read set to the previously reported $L$. rosalia reference mitogenome (NC_021952). We found 4585 matching reads with a mean length of 1104 bp. However, the alignment revealed a high number of SNPs, gaps, 3' artifacts, and low fidelity to the existing reference. Our resulting contig had $96.83 \%$ identity across only $92 \%$ of the query length to $L$. rosalia NC_021952, quite unexpectedly divergent for members of the same species. An unusually large number of reads were mapped to a small, disconnected fragment on the $3^{\prime}$ end of this reference (Fig. S1). To determine whether these were sequencing artifacts or errors in the reference, we aligned our reads to the closely related $L$. chrysopygus mitogenome (accession NC_037878) [23] which yielded fewer reads (2810) of shorter average length (708 bp) but much more uniform coverage. Our

Table 1 Mapping statistics for Control and Adaptive Sampling Runs

\begin{tabular}{|c|c|c|c|c|c|c|}
\hline & \multicolumn{2}{|c|}{$\begin{array}{l}\text { Control run }(1 \mathrm{~h}) \text { on total fecal DNA, } \\
\text { no enrichment }\end{array}$} & \multicolumn{2}{|c|}{$\begin{array}{l}\text { Adaptive Sampling for } 24 \mathrm{~h} \text {, enriching } \\
\text { to S. imperator scaffold genome }\end{array}$} & \multicolumn{2}{|c|}{$\begin{array}{l}\text { 2nd } 24 \mathrm{~h} \text { run enriching to } S \text {. imperator } \\
\text { scaffold }+L \text {. chrysopygus mitogenome }\end{array}$} \\
\hline & $\begin{array}{l}\text { Total } \\
\text { Reads }\end{array}$ & $\begin{array}{l}\text { Alignments to } L \text {. } \\
\text { chrysopygus mitogenome }\end{array}$ & Total Reads & $\begin{array}{l}\text { Alignments to } L \text {. } \\
\text { chrysopygus mitogenome }\end{array}$ & Total Reads & $\begin{array}{l}\text { Alignments to } L \text {. } \\
\text { chrysopygus mitogenome }\end{array}$ \\
\hline $\begin{array}{l}\text { Number of } \\
\text { Sequences }\end{array}$ & 133,852 & 18 & $15,346,306$ & 2810 & $13,362,519$ & 2448 \\
\hline Total Length (bp) & $164,604,258$ & 25,700 & $6,308,923,013$ & $1,990,083$ & $5,683,370,590$ & $3,187,574$ \\
\hline $\begin{array}{l}\text { Average Length } \\
\text { (bp) }\end{array}$ & 1230 & 1428 & 411 & 708 & 425 & 1302 \\
\hline $\begin{array}{l}\text { Mb aligned to } S \text {. } \\
\text { imperator }\end{array}$ & 649,354 & & $50,352,101$ & & $46,619,227$ & \\
\hline $\begin{array}{l}\text { \% Aligned to } S . \\
\text { imperator }\end{array}$ & $0.39 \%$ & & $0.80 \%$ & & $0.82 \%$ & \\
\hline $\begin{array}{l}\text { MtDNA hits per } \\
\text { Mb }\end{array}$ & & 0.11 & & 0.45 & & 0.43 \\
\hline $\begin{array}{l}\text { mtDNA Mb / } \\
\text { Total Mb }\end{array}$ & & 0.016 & & 0.032 & & 0.056 \\
\hline $\begin{array}{l}\text { mtDNA to host } \\
\text { nuclear DNA }\end{array}$ & $3.96 \%$ & & $3.95 \%$ & & & $6.84 \%$ \\
\hline
\end{tabular}


resulting contig had $99.86 \%$ identity over $>99 \%$ of the length of this mitogenome without gaps, suggesting it would provide a better reference for further analyses.

\section{Mitochondrial DNA is enriched by adaptive sampling}

To determine whether adaptive sampling enriched mitochondrial DNA along with nuclear DNA, we compared reads from a non-adaptively sampled control run vs. the first $24 \mathrm{~h}$ of an adaptive sampling run targeting the $S$. imperator scaffold genome. We found a 4-fold increase in number of mtDNA sequences per $\mathrm{Mb}$ when mapping to the $L$. chrysopygus mitogenome from the $S$. imperator enriched reads (Table 1). By read percentage, host mtDNA doubled from 0.016 to $0.032 \%$ of the total fecal DNA, precisely mirroring the doubling seen in total host genomic DNA enrichment. Nanopore adaptive sampling revealed no differences between mitochondrial and host nuclear DNA ratio, with mtDNA making up 3.95\% of the host DNA in both. This result was expected since we enriched for an entire tamarin genome, not just mtDNA specifically.

\section{Higher quality targets increase enriched read length but not sampling efficiency}

Next, we explored whether adaptive sampling efficiency could be improved by adding a more accurate target for enrichment. The S. imperator genome initially used for adaptive sampling contains fragments covering the entire $S$. imperator mitogenome but with very poor quality. When assembled and compared against the high-quality $L$. chrysopygus mitogenome, the two share only $\sim 70 \%$ sequence identity, indicating many errors. In contrast, L. chrysopygus matches 95\% to the existing L. rosalia mtDNA (NC_021952) and > $99 \%$ to the L. rosalia mitogenome that we ultimately assembled. We reasoned that inclusion of the L. chrysopygus mitogenome along with the $S$. imperator full genome might improve adaptive sampling efficiency since it would share much higher sequence identity with $L$. rosalia mtDNA. A second aliquot of the same library was run on the MinION for an additional 24 $\mathrm{h}$, with both the $S$. imperator scaffold genome and $L$. chrysopygus mitogenome as enrichment targets, resulting in $5.6 \mathrm{~Gb}$ of additional reads.

In the second run, reads matching the L. chrysopygus mitogenome were $1302 \mathrm{bp}$ in length, double the $708 \mathrm{bp}$ read length of initial run and is a result of the improved matching to the more closely related and better quality $L$. chrysopygus mitogenome target. This improved length did not result in greater overall enrichment, however, as enrichment remained at 4-fold increase over background. Increased read length did result in $60 \%$ greater coverage of the mitogenome, improving contig coverage from 106x in the first run to $179 \mathrm{x}$ in the second run. Read length improvement and coverage is illustrated in Fig. 1. The region of lowest coverage was the D-loop, while the nearby large subunit rRNA had the highest average coverage. Interestingly, in the second run the ratio of mtDNA to host nuclear DNA nearly doubled from 3.95 to $6.83 \%$ due to the increased read length from having a more accurate mitogenome target.

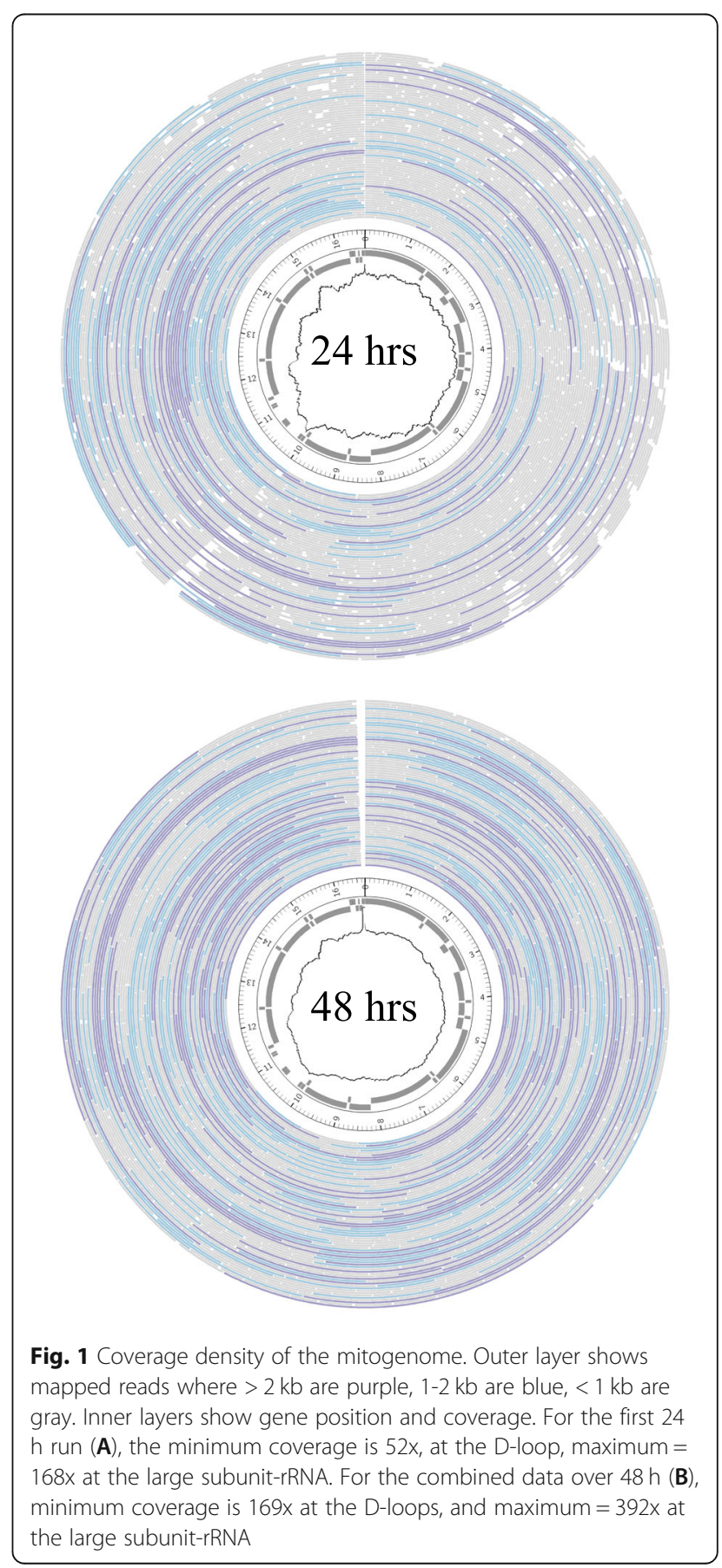




\section{Nanopore adaptive sampling tolerates large target enrichment sequence divergence}

Both rounds of adaptive sampling resulted in 4-fold improvement in mitogenome coverage vs. non-targeted sequencing, in line with Oxford Nanopore's guidance. Since there was only $70 \%$ homology between the available S. imperator mtDNA assembly fragments and our reconstructed $L$. rosalia mitogenome, that indicates adaptive sampling can tolerate at least 30\% sequence identity divergence from the target while still enriching as highly as compared to a perfectly matching target. For subsequent analyses, we combined reads from both runs.

\section{Mapping reveals high coverage of host mitochondrial DNA}

Overall, there were 5516 reads aligning to L . chrysopygus mtDNA with a mean length of $984 \mathrm{bp}$, resulting in $285 \mathrm{x}$ read coverage with improved continuity particularly at the 3' end (Fig. S1). Assembly of reads with Flye yielded a circular draft contig of $16,592 \mathrm{bp}$ with $99.86 \%$ identity to $L$. chrysopygus over $99 \%$ of its length. This draft assembly contained several indels proximal to homopolymeric regions as seen in other reports of unpolished nanopore-generated contigs, suggesting polishing could improve the sequence quality [24]. When our unpolished contig was compared to the existing $L$. rosalia (NC_021952) reference our assembly matched at only $96.83 \%$ identity for $92 \%$ of its length, representing a strong improvement in both measures. The next nearest BLAST match in the NCBI nucleotide database was Goeldi's marmoset (Callimico goeldii) at $85.18 \%$ identity with $97 \%$ query length coverage.

\section{Polishing of the assembly}

Identification of open reading frames was performed with MITOS2 and revealed that the indels in the draft assembly were causing multiple frameshift mutations. For polishing, both the Oxford Nanopore tool Medaka and the 3rd party tool Nanopolish were compared with the former outperforming the latter. Medaka eliminated all frameshifts and was used for the final assembly. Comparison of the draft and polished assemblies revealed 5 indels all proximal to homopolymeric regions and 1 SNP, with identity to L. chrysopygus dropping the match to $99.63 \%$ for $>99 \%$ of its length. This is a substantial improvement compared to the existing $L$. rosalia reference which matched to the polished contig at $95.95 \%$ identity for $95 \%$ of its length. MITOS 2 annotation of the polished assembly found that all frameshift mutations were resolved, and no manual intervention was required. The polished assembly was rearranged to place $C O X 1$ at the start position and submitted to NCBI under accession number MZ262294.

\section{Mitogenome organization is highly conserved with mutations preferentially in the D-loop}

The mitogenome of $L$. rosalia has a total length of $16,597 \mathrm{bp}$ (Fig. 2). Our assembly is shorter than the previous reference by $275 \mathrm{bp}$, and is very similar in size to the closely related L. chrysopygus (16,618 bp) [23]. Coding regions are nearly identical to L. chrysopygus with 2 amino acid changes in $N A D H$ and none in any other. Gene order is the same as in humans and other primates. We then investigated mutations that accrued since the divergence of $L$. rosalia and L. chrysopygus. A total of 38 SNPs are mapped on Fig. 2. As expected, the majority of SNPs are concentrated within the noncoding D-loop. Only 16 SNPs are located throughout the rest of the genome.

\section{DNA methylation is negligible in mitochondria at CpG sites}

Native genomic DNA contains modifications of interest in epigenetic analyses. We chose a sequencing library preparation kit that excludes PCR steps in order to preserve these modifications for downstream analyses. As others have reported both the presence and absence of DNA methylation in mitochondria using a variety of methods, we sought to resolve this controversy by leveraging nanopore's ability to directly detect DNA modifications. First we used the Nanopolish package to detect 5 'methylation $(5 \mathrm{mC})$. Nanopolish was able to call methylation at 259 of the $642 \mathrm{CpG}$ sites in the forward strand only (Fig. 3A). Methylation was called for sites with more than 40 reads (average 160 reads) by Nanopolish. Of these, 29 sites had $>10 \%$ methylation and only 3 sites had $>20 \%$ methylation. Nearly three quarters, 189 sites, had $<5 \%$ methylation. Both technical replicates were combined to generate sufficient coverage for basecalling (Table 2).

To validate and expand these findings, we basecalled methylation again using the ONT package Megalodon as it can call both $5 \mathrm{mC}$ and $5 \mathrm{hmC}$ modifications simultaneously. It uses a neural network model trained on modified cytosines in all contexts, whereas Nanopolish uses a hidden Markov model approach that only supports $5 \mathrm{mC}$ and is less accurate on natural samples containing hydroxymethylation [25]. Megalodon called substantially lower $5 \mathrm{mC}$ at all $642 \mathrm{CpG}$ sites in both strands. Due to increased calling efficiency, the first and second 24-h runs were analyzed separately as technical replicates. Across CpG sites within the mitogenome, DNA methylation $>1 \%$ was seen in just 13 of the 642 total sites, and none of these had greater than $0 \%$ in the technical replicate. Only $2 \mathrm{CpG}$ sites were found to have $>5 \%$ methylation, and neither was above $0 \%$ in the technical replicate. Read coverage was 40x and 63x for each replicate respectively (Table 3 ). We found no significant 


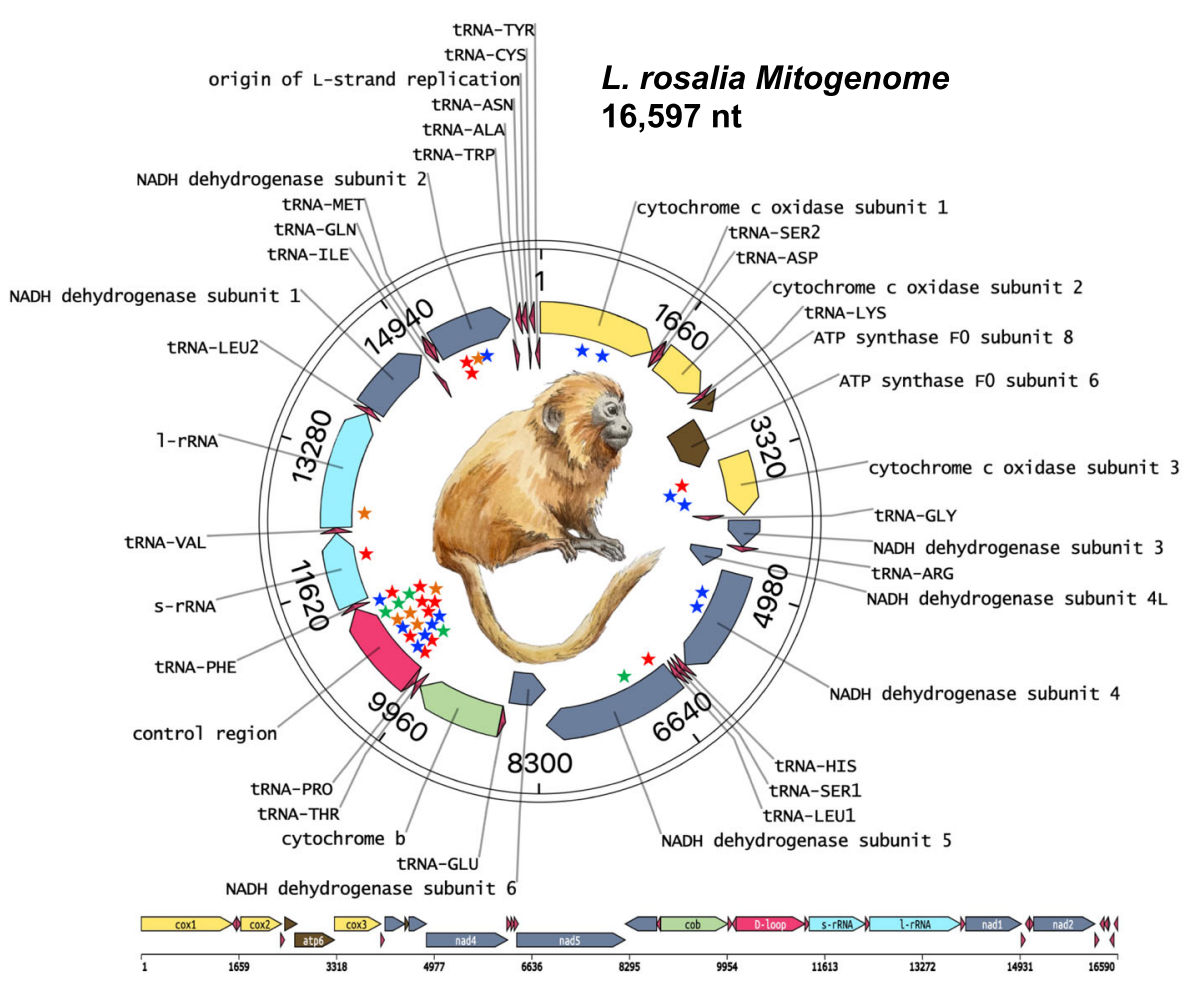

Fig. 2 The mitogenome of the Golden Lion Tamarin. The L. rosalia mitogenome is 16,597 bp in length. Stars represent SNPs in comparison to the closely related Black Lion Tamarin, L. chrysopygus. Star colors indicate base change where red = "T", blue = "C", orange = "G", green = "A". The majority of SNPS are in the D-Loop control region

correlation in between forward and reverse strand methylation levels at any threshold.

\section{DNA hydroxymethylation is moderate at CpG sites}

In contrast to $5 \mathrm{mC}$, mitochondrial hydroxymethylation greater than $1 \%$ was called at nearly half the $642 \mathrm{CpG}$ sites, with 196 of these replicating between runs (Fig. 3B). When filtering for hydroxymethylation greater than $5 \%$, 44 sites were still detected and replicated between runs. At 4 sites $5 \mathrm{hmC}$ exceeded $20 \%$ in both replicates.
Interestingly, $5 \mathrm{hmC}$ is concentrated on the 3 ' side of several genes including COX1, COX3, NAD4, and NAD5 indicating a non-uniform distribution and likely biological function.

\section{Cytosines outside of $\mathrm{CpG}$ context contain no base modifications}

In non-CpG context, identified by the dinucleotide ambiguity code $\mathrm{CH}$ where $\mathrm{H}$ stands for "any nucleotide except G", no cytosines were methylated in any of the

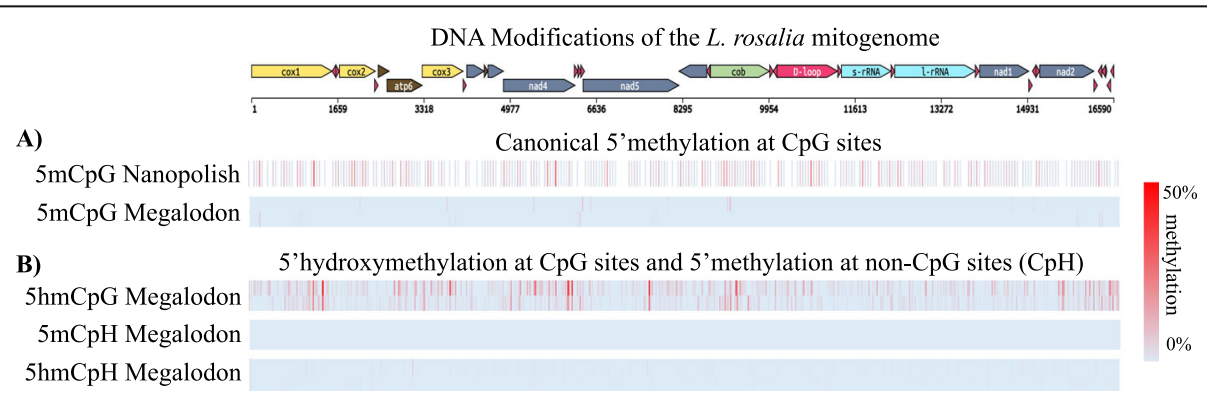

Fig. 3 DNA modifications of the L. rosalia mitogenome. Cytosine modifications are shown mapped to the mitogenome of L. rosalia. A) $5^{\prime}$ methylation is shown by Nanopolish and Megalodon. B) $5^{\prime}$ hydroxymethylation at CpG and CH sites is shown with Megalodon. Each lane is illustrated with replicates at top and bottom from the first and second runs respectively. Hydroxymethylation in the CpG context has the highest prevalence and replication. Percentage of total modified base reads are shown from blue to red 
Table 2 DNA 5'methylation by Nanopolish

\begin{tabular}{lllllll}
\hline \multirow{5}{*}{ m C CpG } & \multicolumn{3}{l}{ Methylation } & & & \\
\cline { 2 - 7 } & Total Sites & $\mathbf{> 1 \%}$ & $\mathbf{> 5 \%}$ & $\mathbf{> 1 0 \%}$ & $\mathbf{> 2 0 \%}$ & Coverage \\
\hline 259 & 189 & 68 & 29 & 3 & $160 x$ \\
\hline
\end{tabular}

5469 possible $\mathrm{CH}$ sites. For hydroxymethylation, we found $3.4 \%$ of sites with detectible levels above $1 \%$, however, only 18 of these sites replicated. At the 5\% threshold, no replication remained. Taken together these findings indicated strong support for the presence of hydroxymethylation in the CpG context, with neither methylation nor hydroxymethylation present outside of CpG sites. (Supplementary File 1).

\section{Discussion}

The purpose of this study was to selectively enrich and sequence host DNA from a non-invasively collected sample using nanopore adaptive sequencing, assemble a mitogenome, and assess for epigenetic modifications. We chose the golden lion tamarin, L. rosalia, as it represents an endangered primate with an unsequenced genome and a mitochondrial genome in need of improvement. The fraction of host DNA sequenced from feces was less than $1 \%$ of the total reads in line with other studies [26], but this fraction was doubled with the use of adaptive sampling. Remarkably, our coverage of the mitogenome was high enough to accurately assemble a full length contig. These findings suggest that mitogenomes make the best target for nanopore sequencing from fecal DNA.

Recently, the mitogenome of the related Brazilian buffy-tufted-ear marmoset (Callithrix aurita) was assembled by genomic skimming with a nanopore sequencer [27]. Genomic skimming uses low coverage sequencing and leverages naturally high copy number sequences (e.g. repeats, and mitogenomes) to assemble high coverage of those regions. It had high concordance with a Sanger sequenced replicate, however it resulted in only $9 \mathrm{x}$ coverage of the mitogenome and required two full flow cells as opposed to our $285 x$ coverage with a single flow cell. Closer to our target species, de Freitas et al. recently used short-read sequencing to generate a high quality mitogenome of L. chrysopygus with over 3000x coverage, providing us with an accurate reference for our nanopore adaptive sampling method [23]. That mitogenome required bootstrapping alignments with manual k-mer adjustments, whereas our long-read pipeline assembled an error-free mitogenome despite lower coverage. Both methods leverage the ability to use related species as alignment targets.

The enrichment of mitochondrial DNA was surprising despite its overabundance compared to nuclear chromosomes. Generally, the ratio of mtDNA to nuclear DNA is about $0.1 \%$, despite containing hundreds to thousands more copies per cell in most somatic tissues [28]. Here we found a 40 -fold increase in this ratio, at 3.96\% mtDNA to nuclear DNA. By including an accurate copy of the L. chrysopygus mitogenome, we increased that ratio to $6.84 \%$, doubled the length of aligned reads, and quadrupled the hits per megabase of reads. Our increased efficiency may be a product of the sample source. Bulk stool contains highly fragmented digested DNA, and the described method may not hold for less degraded mitochondrial DNA. Nanopores draw in only linearized DNA, therefore any mtDNA molecules must be degraded enough to lose their native circular conformation before sequencing. Even though we did not use any double strand break treatments prior to sequencing (e.g. enzymatic treatment, sonication, hydrodynamic shearing), we still sequenced 285 -fold coverage of the mitogenome, as compared to less than $0.1 \mathrm{x}$ of the nuclear genome.

Oxford Nanopore guidance indicates that longer targets and read lengths are correlated with higher enrichment. Our lower level of enrichment (4-fold) over nonenriched sequencing is likely due to short fragment lengths. Indeed, when sequencing human mitochondrial DNA from high molecular weight libraries from liver cells, Goldsmith et al. found reads averaging over $80 \%$ of the entire mitogenome [6]. Meanwhile, adaptive sampling yields best results with targets greater than $15 \mathrm{~kb}$ and read lengths greater than $10 \mathrm{~kb}$ [12]. A second reason for our low level of enrichment was the poor match of the emperor tamarin mtDNA sequences. With the better $L$. chrysopygus match our aligned reads lengthened to $1300 \mathrm{bp}$, slightly longer than the average read length of the control run with no rejection taking place.

Table 3 DNA modification summary by Megalodon

\begin{tabular}{|c|c|c|c|c|c|c|c|c|c|}
\hline & \multirow[b]{2}{*}{ Total Sites } & \multicolumn{3}{|c|}{$>1 \%$ methylation } & \multicolumn{3}{|c|}{$>5 \%$ methylation } & \multicolumn{2}{|c|}{ Coverage } \\
\hline & & 1 st run & 2nd run & \# replicated & 1 st run & 2nd run & \# replicated & 1 st run & 2nd run \\
\hline $5 \mathrm{mC} C \mathrm{pG}$ & 642 & 11 & 13 & 0 & 2 & 2 & 0 & $43 x$ & $63 x$ \\
\hline $5 \mathrm{hmC} \mathrm{CpG}$ & 642 & 328 & 309 & 196 & 124 & 67 & 44 & $43 x$ & $63 x$ \\
\hline $5 \mathrm{mC} \mathrm{CH}$ & 5469 & 0 & 0 & 0 & 0 & 0 & 0 & $49 x$ & $73 x$ \\
\hline $5 \mathrm{hmC} \mathrm{CH}$ & 5469 & 183 & 189 & 18 & 4 & 0 & 0 & $49 x$ & $73 x$ \\
\hline
\end{tabular}


This result indicates that DNA fragmentation in the feces was the limiting factor.

The presence of mitochondrial pseudogenes in the nuclear genome did not appear to bias our results, given the strong concordance of our assembly to the L. chrysopygus mtDNA which was created with careful emphasis to eliminating nuclear coded mt-pseudogenes [23]. The coding structure of our assembly mirrors the L. chrysopygus, with SNPs primarily in the D-loop. SNP accumulation in the D-loop was expected due to its non-coding status.

Our full sequence of the $L$. rosalia mitogenome shares $99.63 \%$ sequence identity with the published $L$. chrysopygus mitogenome, indicating very recent divergence of these sister species. Moreover, the assembly fits better with the known phylogenetic distances within Callitrichidae. The sister taxa relationship between these species had been under debate until 2001 when photoreceptor intron sequences unambiguously established their relationship [21]. In, 2008 the first mitochondrial sequences, of the D-loop, confirmed their close relationship [7]. We extend this evidence by showing near perfect synteny throughout the coding regions.

The presence of mitochondrial DNA modifications has long been debated. Here we report essentially zero DNA methylation at every $\mathrm{CpG}$ position in the mitogenome in stark contrast to other reports of low but consistent methylation in mtDNA. Mitochondrial methylation using a nanopore sequencer has been reported by at least four groups [6,29-31]. All of these studies found low but measurable mtDNA methylation, averaging less than $7 \%$ across a variety of conditions and cell types, though a few specific CpG sites had over 20\%. These studies all used Nanopolish which does not distinguish $5 \mathrm{mC}$ from $5 \mathrm{hmC}$. Since bisulfite-based methods also cannot distinguish $5 \mathrm{mC}$ from $5 \mathrm{hmC}$, they are similarly prone to overestimating the level of $5 \mathrm{mC}$. It is also well-known that bisulfite does not transform circular DNA with high efficiency, again leading to an overestimate of $5 \mathrm{mC}$ [32]. Nanopore alleviates these concerns by avoiding bisulfite conversion, and all DNA molecules sequenced through a pore are linearized by necessity. Here we used Megalodon's neural network model capable of detecting $5 \mathrm{hmC}$ to avoid Nanopolish's $5 \mathrm{mC}$ bias. In support of our findings, a recent careful analysis of mitochondria methylation by Bicci et al. validated nanopore sequencing across multiple primary and cancer cell lines [30]. When accounting for all confounders, they found negligible DNA methylation as we have here. We suggest that previous findings of mitochondrial $5 \mathrm{mC}$ were artefacts of either bisulfite or miscalled nanopore basecalling.
We found very high levels of 5 'hydroxymethylation within the mitogenome in the CpG context. Interestingly, the most well-cited work on mitochondrial base modifications by Shock et al. also reported 10x greater levels of $5 \mathrm{hmC}$ than $5 \mathrm{mC}$, using antibody-based methods [33]. Biological function of 5 'hydroxycytosine is strongly suggested for two reasons. First, we found no non-CpG modifications at all. This argues for reader proteins capable of recognizing cytosines in dinucleotide context rather than these modifications resulting from non-specific oxidation processes modifying cytosines by chance. Interestingly we saw no correlation in $5 \mathrm{hmC}$ between strands, indicating a non-palindromic oxidation process. This stranded-ness in mitochondrial base modification was seen by Dou et al., though they used bisulfite methods and called it as $5 \mathrm{mC}$ [34]. Second, we see enrichment on the 3 ' side of several genes, showing non-uniform distribution which is a hallmark of function [35]. Taken together, our data suggests a specific biological function of $5 \mathrm{hmCpG}$ in mitochondria.

Epigenetic data is challenging to recover from wild species but has been done in hyenas and is expanding to other vertebrates [36, 37]. Therefore, our study serves two purposes. First, it confirms the ability to detect base modifications by nanopore sequencing of mitogenomes [14]. Second, it provides proof-ofconcept that epigenetic analyses can be performed on fecal derived samples, which is applicable to both host nuclear genome methylation and the fecal microbiome, even potentially including food species in digesta.

It is important to consider some caveats to our method. Nanopore accuracy is still low relative to shortread and Sanger sequencing, though neural network models are improving at a rapid pace [15]. Polishing helps as indicated by Medaka's ability to removing all frame shift inducing indels from our assembly. Modified base calling is also improving rapidly and is also dependent on models trained preferably for a specific modification and with taxa-specific data.

Interest in long-read sequencing of vertebrate mitogenomes continues to increase. A study by Formenti et al. recently sampled 100 species and corrected many long-standing errors in reference mitogenomes [38]. However, their approach is unsuitable for the most easily available tissue, fecal DNA, since the first step is to remove short fragments less than $10 \mathrm{~kb}$. Here we show the strength of short fragments and long-read technology together to generate accurate assemblies and epigenomes useful for comparative genomics [39]. Accurate genomic resources are critical to the conservation of endangered species like the Golden Lion Tamarin. 


\section{Materials and methods Sample collection}

A single fecal sample weighing $10 \mathrm{~g}$ was collected 10 min post defecation from the environment of the two golden lion tamarins at the Utica Zoo The sample was witnessed as being deposited by the female of a pair, Arie, who was 6 years old at the time of collection. The sample was collected under the approval of the Utica zoo. Fecal samples are exempt from IACUC protocol approval at the University of Minnesota.

\section{DNA extraction}

Qiagen QIAamp DNA stool mini kit (cat no. 51504) was used with the protocol, "Isolate of DNA from Stool for $\mathrm{Hu}$ man DNA Analysis" supplied by manufacturer. This kit yielded approximately $4 \mu \mathrm{g}$ of purified DNA from $200 \mathrm{mg}$ of fecal sample. Additionally, the Omega Bio-tek E.Z.N.A. Stool DNA kit (cat no. D4015-00) was used also with $200 \mathrm{mg}$ of stool and manufacturer's protocol, "DNA Extraction and Purification from Stool for Human DNA Detection" and yielded a similar quantity of DNA. We used two kits since the Qiagen kit is no longer manufactured and its replacement is twice the price of the Omega Bio-tek kit which yielded similar quantity and quality.

DNA from both kits were combined and library prep was performed using the Oxford Nanopore Technologies (ONT) SQK-LSK109 "Genomic DNA by Ligation" protocol with the following changes: 1) NEBNext products were used for end-repair as suggested by ONT (cat no. M6630, E7546, and E6056), 2) Axygen AxyPrep Mag PCR Clean-up beads were substituted for Agencourt AMPure beads, 3) magnetic beads were optionally diluted to $25 \%$ of original volume with in-house prepared carboxy bead dilution buffer (https://bomb.bio/ protocols/) for cost savings with no loss in recovery efficiency [40].

\section{Nanopore adaptive sequencing}

Sequencing was conducted on a single MinION sequencer on a FLO-min106 flow cell with pore chemistry R9.4 for two runs of $24 \mathrm{~h}$ each. Prior to the first run, the cell was run for $1 \mathrm{~h}$ without adaptive sampling, providing control data. Between $24 \mathrm{~h}$ runs the flow cell was washed with nuclease from the Flow Cell Wash Kit (WSH004) and loaded with storage buffer. A second aliquot from the same initial library prep was used for the second run. Live basecalling was performed using the fast basecalling model in ONT basecalling software, Guppy v4.5.4, GPU enabled with a GeForce RTX 2080Ti. Adaptive sampling was enabled in MinKNOW core v.4.2.5 and set to enrich sequences matching to the Saguinus imperator (emperor tamarin) genome, SagImp_v1_BIUU (accession PRJNA399417), scaffold assembly. After sequencing, reads were basecalled again with Guppy using the high accuracy model and these reads were used for all subsequent analyses. Basecalled reads were mapped using Minimap2 [41] to the L. rosalia (NC_021952) and L. chrysopygus (NC_037878) mitogenomes. Resulting bam files were sorted and indexed using samtools and converted to bed files for viewing in IGV with bamToBed from bedtools [42, 43]. Visualization of read statistics was performed with Bamstats (http://bamstats. sourceforge.net).

\section{Mitogenome assembly}

Assembly proceeded in two steps. We used the total reads aligning to L. chrysopygus mtDNA in fasta format to generate the assembly. Flye v2.8.3 was used for draft assembly and first round polishing of the initial consensus [44]. Flye has an expected error rate of 0.5 to $1 \%$ for ONT reads and therefore was followed by an additional polishing step. Medaka v1.3.2 uses neural network models applied to a pileup of individual sequencing reads against a draft assembly to improve consensus sequences. In our testing, it outperformed Nanopolish in generating a contig without frameshift indels in the final contig [25]. Both packages were used with default settings.

\section{Circularization and annotation}

Flye generated a circularized assembly, i.e. no repeats on the ends, ready for annotation. Identification of protein coding regions was performed through the MITOS2 website (http://mitos2.bioinf.uni-leipzig.de). Start position was manually set at the COX1 gene following convention. The genome was visualized in Open Vector Editor (https://github.com/TeselaGen/openVectorEditor) and submitted to NCBI.

\section{DNA methylation analyses}

Nanopolish calls methylation uses raw signal data with a hidden Markov model. The Nanopolish call-methylation function was applied on the same reads used for consensus building. Site-specific methylation percentages were determined using the calculate_methylation_frequency.py helper script.

Megalodon extracts high accuracy modified bases and variant calls from raw nanopore reads by using intermediate output from the neural network model provided by the basecaller Guppy. We used Megalodon v2.3.1 with the Guppy v4.6.4. The following research model from Rerio repository, res_dna_r941_min_modbases_ $5 \mathrm{mC} \_5 \mathrm{hmC}$ _v001.cfg, was used as it is able to call both $5 \mathrm{mC}$ and $5 \mathrm{hmC}$ modifications at cytosine sites in any context. The "--mod-binary-threshold $=0.8$ " flag was set to slightly decrease the stringency of Megalodon's modification calling in line with suggested practices [6]. Further details are in supplementary file 2. 


\section{Abbreviations}

CpG: Cytosine-phosphate-guanine; ONT: Oxford Nanopore Technologies; 5mC: 5'methylcytosine; $5 \mathrm{mCpG}$ : 5'methylation at CpG site;

5hmC: 5'hydroxymethylcytosine; $5 \mathrm{hmCpG}$ : 5'hydroxymethylation at CpG site; $\mathrm{CH}$ : Cytosine plus any non-G base

\section{Supplementary Information}

The online version contains supplementary material available at https://doi. org/10.1186/s12864-021-08046-7.

Additional file 1: Fig. S1. Alignment of reads to $L$. rosalia from the first $24 \mathrm{~h}$ run to the reference mitogenome (KC757399) has $96.83 \%$ contig identity over $92 \%$ of its length, with $5^{\prime}$ gaps (top). Alignment to $L$. chrysopygus reference (NC_037878) improves to 99\% with complete coverage over the full length (bottom). The 5516 reads are combined from 2810 (1st run) and 2706 (2nd run)

Additional file 2: Supplementary File 1. Supplementary Methods. Additional file 3: Table S7. 5' methylcytosine CpG calls from Nanopolish.

Additional file 4: Supplementary File 3. Consensus sequence of $L$. rosalia mitogenome in fasta format.

Additional file 5: Supplementary File 4. Alignment reads used for mitogenome assembly.

\section{Acknowledgements}

We thank Executive Director Andria Heath, Director of Administrative Operations Nikki Sheehan and the keeper and animal care staff of the Utica Zoo as well as their tamarins Arie and Kane for generous donation of fecal material. We also thank A. Barks for experimental suggestions with DNA methylation analysis. We thank Sydney McGraw (sydneyruthart@gmail.com) for the tamarin drawing. The authors have no conflicts of interest and declare no competing financial interests.

\section{Data access}

The mitogenome sequence has been deposited into NCBI GenBank with accession number MZ262294 and is available as supplementary file 3. Reads used to generate this assembly are available as supplementary file 4.

\section{Authors' contributions}

CF conceived and designed the study. AM obtained the samples. CF performed library prep and sequencing. NW, CF, and PL analyzed and interpreted the data. NW, AM, and CF drafted the manuscript. All authors reviewed and approved the manuscript.

\section{Funding}

This work was supported by USDA AES Project. No. MIN-16-129, the UM Informatics Institute, and the SUNY Polytechnic Institute Research Foundation.

\section{Availability of data and materials}

The mitogenome sequence has been deposited into NCBI GenBank with accession number MZ262294 and is available as supplementary file 3. Reads used to generate this assembly are available as supplementary file 4. Prior tamarin sequences were sourced from NCBI Genbank which is an open public access repository [https://www.ncbi.nlm.nih.gov/genbank].

\section{Declarations}

\section{Ethics approval and consent to participate}

Primate fecal samples were collected under the institutional approval of the Utica zoo (Utica, NY). Fecal samples are exempt from IACUC protocol approval at the University of Minnesota.

\section{Consent for publication}

NA

\section{Competing interests}

The authors have no competing interests to declare.

\section{Author details}

Department of Animal Sciences, University of Minnesota, College of Food Agricultural, and Natural Resource Sciences, 1988 Fitch Ave., Saint Paul, MN 55108, USA. ${ }^{2}$ Department of Veterinary and Biomedical Sciences, College of Veterinary Medicine, University of Minnesota, Saint Paul, MN, USA.

${ }^{3}$ Department of Biology and Chemistry, College of Arts and Sciences, SUNY Polytechnic Institute, Utica, NY, USA.

Received: 9 August 2021 Accepted: 29 September 2021

Published online: 07 October 2021

\section{References}

1. Rylands $A B$, Kierulff MCM, de Souza Pinto LP. Distribution and status of lion tamarins. In: Kleiman DG, Rylands AB, editors. Lion Tamarins: biology and conservation. 1st ed. Washington, D.C.: Smithsonian Institution Press; 2002. p. 42-70.

2. Groves CP. Primate Taxonomy. 1st ed. Washington, D.C.: Smithsonian Institution Press; 2001.

3. Kierulff MCM, Ruiz-Miranda CR, de Oliveira PP, Beck BB, Martins A, Dietz JM, et al. The Golden lion tamarin Leontopithecus rosalia: a conservation success storyTitle. Int Zoo Yb. 2012;46(1):36-45. https://doi.org/10.1111/j.174 8-1090.2012.00170.x

4. Coimbra-Filho AF, Mittermeier RA. Distribution and ecology of the genus Leontopithecus lesson, 1840 in Brazil. Primates. 1973;14(1):47-66.

5. Lapenta MJ, Procópio-de-Oliveira P, Kierulff MCM, Motta-Junior J. Frugivory and seed dispersal of golden lion tamarin (Leontopithecus rosalia (Linnaeus, 1766)) in a forest fragment in the Atlantic Forest, Brazil (Frugivoria e dispersão de sementes por Micos-Leões-Dourados) (Leontopithecus rosalia) em um fragmento florest. Braz J Biol. 2008;68(2):241-9. https://doi.org/10.1 590/S1519-69842008000200004.

6. Goldsmith C, Rodríguez-Aguilera JR, El-Rifai I, Jarretier-Yuste A, Hervieu V, Raineteau O, Saintigny P, Chagoya de Sánchez V, Dante R, Ichim G, Hernandez-Vargas H. Low biological fluctuation of mitochondrial CpG and non-CpG methylation at the single-molecule level. Sci Rep. 2021;11:8032.

7. Perez-Sweeney BM, Valladares-Padua C, Martins CS, Morales JC, Melnick DJ. Examination of the taxonomy and diversification of Leontopithecus using the mitochondrial control region. Int J Primatol. 2008;29(1).

8. Matauschek C, Roos C, Heymann EW. Mitochondrial phylogeny of Tamarins (Saguinus, Hoffmannsegg 1807) with taxonomic and biogeographic implications for the s. nigricollis species group. Am J Phys Anthropol. 2011; 144(4).

9. Finstermeier K, Zinner D, Brameier M, Meyer M, Kreuz E, Hofreiter M, et al. A Mitogenomic Phylogeny of Living Primates. PLoS One. 2013;8(7).

10. Lu H, Giordano F, Ning Z. Oxford Nanopore MinION sequencing and genome assembly. Vol. 1e4, Genomics, Proteomics and Bioinformatics. 2016

11. Gilpatrick T, Lee I, Graham JE, Raimondeau E, Bowen R, Heron A, et al. Targeted nanopore sequencing with Cas9-guided adapter ligation. Nat Biotechnol. 2020;38(4).

12. Payne A, Holmes N, Clarke T, Munro R, Debebe BJ, Loose M. Readfish enables targeted nanopore sequencing of gigabase-sized genomes. Nat Biotechnol. 2020;

13. Sharma N, Pasala MS, Prakash A. Mitochondrial DNA: Epigenetics and environment. Vol. 60, Environmental and Molecular Mutagenesis. 2019

14. Chandler J, Camberis M, Bouchery T, Blaxter M, Le Gros G, Eccles DA. Annotated mitochondrial genome with Nanopore R9 signal for Nippostrongylus brasiliensis. F1000Research. 2017;6.

15. Wick RR, Judd LM, Holt KE. Performance of neural network basecalling tools for Oxford Nanopore sequencing. Genome Biol. 2019;20(1).

16. Xiao C Le, Zhu S, He M, Chen D, Zhang Q, Chen Y, et al. N 6 -Methyladenine DNA Modification in the Human Genome. Mol Cell. 2018; $71(2)$

17. Wang Z, Zolnik CP, Qiu Y, Usyk M, Wang T, Strickler HD, et al. Comparison of fecal collection methods for microbiome and metabolomics studies. Front Cell Infect Microbiol. 2018;8(AUG).

18. Cuscó A, Salas A, Torre C, Francino O. Shallow metagenomics with Nanopore sequencing in canine fecal microbiota improved bacterial taxonomy and identified an uncultured CrAssphage. bioRxiv. 2019.

19. Moss EL, Maghini DG, Bhatt AS. Complete, closed bacterial genomes from microbiomes using nanopore sequencing. Nat Biotechnol. 2020;38(6). 
20. Shanmuganandam S, Hu Y, Strive T, Schwessinger B, Hall RN. Uncovering the microbiome of invasive sympatric European brown hares and European rabbits in Australia. bioRxiv. 2019.

21. Mundy NI, Kelly J. Phylogeny of lion tamarins (Leontopithecus spp) based on interphotoreceptor retinol binding protein intron sequences. Am J Primatol. 2001;54(1).

22. Rosenberger AL, Coimbra-Filho AF. Morphology, Taxonomic Status and Affinities of the Lion Tamarins, Leontopithecus (Callitrichinae, Cebidae). Folia Primatol. 2008;42(3-4)

23. de Freitas PD, Mendez FL, Chávez-Congrains K, Galetti PM, Coutinho LL, Pissinatti $A$, et al. Next-generation sequencing of the complete mitochondrial genome of the endangered species Black Lion Tamarin Leontopithecus chrysopygus (primates) and mitogenomic phylogeny focusing on the callitrichidae family. G3 Genes, Genomes, Genet. 2018; 8(6).

24. Baeza JA. Yes, we can use it: a formal test on the accuracy of low-pass nanopore long-read sequencing for mitophylogenomics and barcoding research using the Caribbean spiny lobster Panulirus argus. BMC Genomics. 2020;21(1).

25. Simpson JT, Workman RE, Zuzarte PC, David M, Dursi LJ, Timp W. Detecting DNA cytosine methylation using nanopore sequencing. Nat Methods. 2017; 14(4):407-10. https://doi.org/10.1038/nmeth.4184.

26. Sharma AK, Pafčo B, Vlčková K, Červená B, Kreisinger J, Davison S, et al. Mapping gastrointestinal gene expression patterns in wild primates and humans via fecal RNA-seq. BMC Genomics. 2019;20(1).

27. Malukiewicz J, Cartwright RA, Dergam JA, Igayara CS, Nicola PA, Pereira LMC, et al. Genomic Skimming and Nanopore Sequencing Uncover Cryptic Hybridization in One of World's Most Threatened Primates. bioRxiv [Internet]. 2021 Jan 1;2021.04.16.440058. Available from: http://biorxiv.org/ content/early/2021/04/17/2021.04.16.440058.abstract

28. Robin ED, Wong R. Mitochondrial DNA molecules and virtual number of mitochondria per cell in mammalian cells. J Cell Physiol. 1988;136(3)

29. Aminuddin A, Ng PY, Leong CO, Chua EW. Mitochondrial DNA alterations may influence the cisplatin responsiveness of oral squamous cell carcinoma. Sci Rep. 2020;10(1).

30. Bicci I, Calabrese C, Golder ZJ, Gomez-Duran A, Chinnery PF. Oxford Nanopore sequencing-based protocol to detect $\mathrm{CpG}$ methylation in human mitochondrial DNA. bioRxiv. 2021;

31. Lüth T, Klein C, Schaake S, Tse R, Pereira S, Lass J, et al. Analysis of mitochondrial genome methylation using Nanopore single-molecule sequencing. bioRxiv [Internet]. 2021 Jan 1;2021.02.05.429923. Available from: http://biorxiv.org/content/early/2021/02/06/2021.02.05.429923.abstract

32. Mechta $M$, Ingerslev LR, Fabre $O$, Picard $M$, Barrès R. Evidence suggesting absence of mitochondrial DNA methylation. Front Genet. 2017;8(NOV).

33. Shock LS, Thakkar P V., Peterson EJ, Moran RG, Taylor SM. DNA methyltransferase 1, cytosine methylation, and cytosine hydroxymethylation in mammalian mitochondria. Proc Natl Acad Sci U S A. 2011;108(9).

34. Dou X, Boyd-Kirkup JD, McDermott J, Zhang X, Li F, Rong B, et al. The strand-biased mitochondrial DNA methylome and its regulation by DNMT3A. Genome Res. 2019;29(10).

35. McLain AT, Faulk C. The evolution of CpG density and lifespan in conserved primate and mammalian promoters. Aging (Albany NY). 2018;

36. Laubach ZM, Faulk CD, Dolinoy DC, Montrose L, Jones TR, Ray D, et al. Early life social and ecological determinants of global DNA methylation in wild spotted hyenas. Mol Ecol. 2019;

37. De Paoli-lseppi R, Deagle BE, McMahon CR, Hindell MA, Dickinson JL, Jarman SN. Measuring animal age with DNA methylation: From humans to wild animals. Vol. 8, Frontiers in Genetics. 2017.

38. Formenti G, Rhie A, Balacco J, Haase B, Mountcastle J, Fedrigo O, et al. Complete vertebrate mitogenomes reveal widespread gene duplications and repeats. bioRxiv. 2020.

39. Colwell M, Drown M, Showel K, Drown C, Palowski A, Faulk C. Evolutionary conservation of DNA methylation in CpG sites within ultraconserved noncoding elements. Epigenetics [Internet]. 2018;13(1):49-60. Available from:. https://doi.org/10.1080/15592294.2017.1411447.

40. Oberacker P, Stepper P, Bond DM, Höhn S, Focken J, Meyer V, et al. Bio-OnMagnetic-Beads (BOMB): Open platform for high-throughput nucleic acid extraction and manipulation. PLoS Biol. 2019;17(1).

41. Li H. Minimap2: Pairwise alignment for nucleotide sequences. Bioinformatics. 2018;34(18)
42. Li H, Handsaker B, Wysoker A, Fennell T, Ruan J, Homer N, et al. The Sequence Alignment/Map format and SAMtools. Bioinformatics. 2009;25(16).

43. Quinlan AR, Hall IM. BEDTools: A flexible suite of utilities for comparing genomic features. Bioinformatics. 2010;26(6).

44. Kolmogorov M, Bickhart DM, Behsaz B, Gurevich A, Rayko M, Shin SB, et al. metaFlye: scalable long-read metagenome assembly using repeat graphs. Nat Methods. 2020;17(11).

\section{Publisher's Note}

Springer Nature remains neutral with regard to jurisdictional claims in published maps and institutional affiliations.

\section{Ready to submit your research? Choose BMC and benefit from:}

- fast, convenient online submission

- thorough peer review by experienced researchers in your field

- rapid publication on acceptance

- support for research data, including large and complex data types

- gold Open Access which fosters wider collaboration and increased citations

- maximum visibility for your research: over $100 \mathrm{M}$ website views per year

At BMC, research is always in progress.

Learn more biomedcentral.com/submissions 